\title{
Three-dimensional Television: Consumer, Social, and Gender Issues
}

\author{
Haldun M. Ozaktas \\ Bilkent University, TR-06800 Bilkent, Ankara, Turkey
}

This chapter is based on a series of discussions which were planned and carried out within the scope of the Integrated Three-Dimensional TelevisionCapture, Transmission, and Display project, which is a Network of Excellence (NoE) funded by the European Commission 6th Framework Information Society Technologies Programme. The project involves more than 180 researchers in 19 partner institutions from 7 countries throughout Europe and extends over the period from September 2004 to August 2008.

The scope of the discussions encompassed consumer expectations and behavior, including current perceptions of three-dimensional television (3DTV), its potential for novelty and mass consumption, and other consumer and nonconsumer applications and markets for the technology. Other areas discussed included the social dimensions of 3DTV in both consumer and non-consumer spheres, and how it compares with other high-impact technologies. Gender related issues were also discussed to some degree.

Neither the manner in which the discussions were conducted, nor the way in which they were processed, was based on a scientific methodology. All discussions were informal in nature, with the moderator periodically putting up discussion points to raise new issues or focus the discussion.

Most participants in these discussions were technical professionals or academicians with backgrounds in engineering and science, who were members of the Network of Excellence. A number of professionals from other areas also enriched the discussions and a small sample of laypersons and potential consumers were interviewed briefly. Our reporting here by no means represents a sequential record of the live and e-mail discussions which spanned a period of over two years. Opinions provided at different times and places were montaged thematically to achieve a unified presentation and were heavily edited.

The discussions given here may seem naive (or worse, misguided) to social scientists with more sophisticated skills and more experience in thinking about such issues. Our hope is that if the content of this chapter does not actually illuminate the issues within its scope, it may at least shed light on the level of thinking and the concerns of those who are actively developing the technology. 
In this case, we hope that this chapter will serve as an insider record of the ruminations of the developers of a technology about its implications, during an intermediate stage of its development. It will certainly be interesting to consider these in retrospect ten or twenty years from now.

\section{Part I: Introduction}

\subsection{Introduction}

It would certainly be a mistake to look upon three-dimensional television (3DTV) as solely the latest in the line of media technologies from radio to black-and-white television to color television, although therein may lie its greatest economic potential. Complicated chains and networks of causality underly the interaction between many technologies and society. It is important to distinguish between the impacts of social and technological entities, although they are intimately related. Television as a social institution has been thoroughly discussed, generally with a negative tone. Totally different however is the legacy of television in the sense of broadcasting technology, or in the sense of the cathode ray tube (CRT), the central technology in conventional television. This technology has been perfected for consumer television units, but today finds many applications, most notably in computer display terminals. Indeed, it can be argued that the CRT has had a greater impact in computing than in television. (Ironically, the liquid crystal display (LCD) found a place in computing first, and then later in television sets.) Lastly, it is important to make a distinction between 3D displays and 3D television (3DTV). Here we use the term 3D display to refer to imaging devices which create $3 \mathrm{D}$ visual output. 3DTV refers to the whole chain of 3D image acquisition, encoding, transport/broadcasting, reception, as well as display.

We must also be cautious when referring to the impact of a technology on society, as it implies that there is only one-way causation; technology may have an impact on society but society also has an effect on technology. Such considerations complicate any prediction regarding the impact of 3DTV. However it seems very likely that it will have an important impact. Home video, cable, broadcast, and games are potentially highly rewarding areas for earlyentrance companies, since it may take a while before the technology can be emulated by others. Widespread public acceptance of this technology is very difficult to predict and will depend largely on the quality attained. If only mediocre quality is feasible, market penetration may be shallow and short lived, relying more on novelty aspects which are known to wear off quickly. People may prefer a high-quality two-dimensional image to a medium quality three-dimensional one, especially if there are limitations on viewing angle, contrast, equipment size, and cost. Even so, three-dimensional television has been so heavily portrayed in film and fiction that a significant number of consumers may show interest despite possible shortcomings. On the other hand, 
if reasonably high quality can be attained, even at an initially high price, it is possible, and indeed likely that the technology may supplant ordinary television in at least some contexts.

The potential consumer market should not blind one to the opportunities in other more specialized applications. Most of these will not demand as high a quality as consumer applications, and may involve customers willing to pay higher prices. While it is not clear that 3DTV would be widely used for computer display terminals, there are a wide variety of specialized applications. These may include sophisticated computer games, professional simulators and virtual reality systems, teleconferencing, special-purpose applications including scientific and industrial visualization, inspection, and control, medical visualization and remote diagnosis and treatment including telesurgery, environmental monitoring, remote operation in hazardous environments, air traffic control, architectural and urban applications, and virtual preservation of perishable objects of cultural heritage.

If we accept that two-dimensional imaging and display technologies have had a positive impact in modern society, it seems almost certain that the above applications will produce a positive impact, even if $3 \mathrm{DTV}$ does not become a standard item in every home. For instance, the fact that people still travel to meet face-to-face is evidence that even modern teleconferencing cannot fully replace physical proximity. If 3DTV can come close enough, this would have a large impact on how meetings are conducted. This would not only include official and corporate meetings (reducing the cost of products and services to society), but also the meetings of civil society organizations, potentially increasing public participation at all levels.

Three-dimensional television should not be seen in isolation from other trends in media technology, most importantly interactive or immersive technologies. Cliches holding television responsible for the drop in theater attendance or reading will gain new strength if such technologies become widespread. The main question will again focus on what the new technologies will replace/displace.

In summary, the potential applications of the technology fall in two main categories: a three-dimensional replacement of present day television and a variety of specialized applications. The impact of the latter could be moderate to high benefits to society in economic and welfare terms. The impact of the former is less predictable but there is the potential for very high economic returns to those who own the technologies.

\subsection{Historical Perspective}

I. Rakkolainen provided an extended account of pertinent historical observations, summarized at length in this section. He noted that many have dreamed of Holodeck- or StarWars-like 3D displays and that 3D images have attracted 
interest for over a century. The general public was excited about 3D stereophotographs in the 19th century, 3D movies in the 1950s, holography in the 1960s, and is now excited by $3 \mathrm{D}$ computer graphics and virtual reality (VR). The technology of 3D displays has deeply intrigued the media and the public. Hundreds of different principles, ideas, and products have been presented with potential applications to scientific visualization, medical imaging, telepresence, games, and 3D movies. The broad field of VR has driven the computer and optics industries to produce better head-mounted displays and other types of 3D displays; however most such VR efforts involve wearing obtrusive artifacts, an experience in stark contrast with the ease of watching TV. Immersion is an experience that encloses the user into a synthetically generated world. Contemporary 3D displays try to achieve this through elaborate schemes, but this is not only a matter of technology; the most important factor for immersion is not technical fidelity but the user's attitude and possibly the skill of the content author. A theater scene or a novel can be quite "immersive" although it does not involve very advanced technology.

Just before the first photographs were made in 1839, stereo viewing was invented. The first stereoscope tried to reproduce or imitate reality with the aid of an astonishing illusion of depth. A decade later, when less cumbersome viewing devices were developed, stereoscopic photography became popular. The stereo image pairs immersed the viewer in real scenes (they are still popular in the form of toys). Then, starting at the end of the 19th century moving pictures reproduced a world of illusion for the masses.

The idea of synthetically-reproduced reality is not new and does not necessarily rely on digital technology. In 1860 the astronomer and scientist Herschel wrote about his vision of representing scenes in action and handing them down to posterity. Cinema and TV have somewhat fulfilled his vision.

I. Rakkolainen went on to list a large number of popular mass-produced cameras of the late 19th century, each of which used slightly different technologies and designs with no standards: Academy, Brownie, Buckeye, Comfort, Compact, Cosmopolitan, Delta, Eclipse, Filmax, Frena, Harvard, Kamaret, Kodak, Kombi, Lilliput, Luzo, Nodark, Omnigraphe, Photake, Poco, Simplex, Takiv, Velographe, Verascope, Vive, Weno, Wizard, and Wonder. Only Kodak survived and became a huge business. The Kodak camera was by no means a superior technology. It used a roll film long enough for 100 negatives, but the key element to its success was perhaps that Kodak provided a photofinishing service for customers; apparently having to do the lab work was an obstacle for many. Rakkolainen believes that this resembles the current situation with 3DTV. The same enthusiasm that greeted photography, stereographs, and Lumiere brothers' Cinematographe at the end of the 19th century, is now seen with $3 \mathrm{DTV}$, virtual reality, and other related technologies. 


\section{Part II: Consumer Expectations and Behavior}

\subsection{Current Public Perceptions of "Three-dimensional Television"}

\section{What do lay people think of when confronted with the phrase "three-dimensional television"?}

This question was posed to people from different social and educational backgrounds. Among the brief answers collected we note the following:

- People think of the image/scene jumping out, or somehow extending from the front of the screen. Although not everyone had seen Princess Leya projected by R2D2 in Star Wars, the idea of a crystal ball is widespread in folklore. However, most people seem to imagine a vertical display like conventional TV, rather than a horizontal tabletop scenario.

- So-called "three-dimensional" computer games which are not truly threedimensional, but where the action takes place in a three-dimensional domain as opposed to early computer games which take place in "flatland."

- "Nothing."

M. Kunter noted that some thought of 3DTV as a full 3D projection of objects into the room, but nobody referred to the "Holodeck" scenario (being and acting in a virtual reality environment). This may be connected to A. Boev's distinction between what he refers to as convergent and divergent $3 \mathrm{D}$ displays: He defines convergent 3D as the case where the user stays outside the presentation; the presentation can be seen from different points of view, like observing a statue or attending the theater. He defines divergent $3 \mathrm{D}$ as the case where the user is inside the presentation, and is able to look around and change points of view. Boev noted that this is often compared to an immersive multimedia-type of game, and is in some ways like listening to radio theater which also puts the user in a similar state of mind of "being inside" the presentation. The observation that a radio play makes one feel inside, compared to TV where one feels outside, seems very important; the perception of insideness, which is considered an aspect of realism, does not necessarily increase with the amount of information conveyed.

According to I. Rakkolainen, 3DTV may take many different forms; it may be similar to today's TVs but with 3D enhancements, IMAX-like partially immersive home projection screens, tracking head-mounted displays, holographic displays, or perhaps "immaterial" images floating in the air. He emphasized that we should be open minded about the possibilities. A. Boev noted that the very use of the term 3DTV was limiting in that it forced people to think of a box, and excluded other modalities. M. Karamüftüoğlu asked whether 3DTV would be immersive and/or interactive, or merely offer depth information. He also noted that the TV and computer box might disappear, with all such technologies converging to an ubiquitous, pervasive presence. 
M. Özkan told an interesting anecdote exemplifying the power of media and marketing: He had asked TV sales staff in electronics stores whether they had heard about 3DTV and amazingly they said that it was "coming soon." And what they were referring to was not any stereoscopic display, but a device displaying miniature football players on a table-like surface; they had seen it on a TV program featuring the 3DTV NoE! Furthermore, they linked this "near-future product" to recent price cuts in plasma and LCD TV screens. This anecdote is powerful evidence of how certain images can capture the public imagination. H. M. Ozaktas recalled that one US telephone commercial from about ten years ago, showed a family reunion for a child's birthday party taking place through teleconferencing. The image took up a whole wall, making it seem that the remote participants were in the other half of the room. Clearly, the makers of the commercial were trying to similarly capture the imagination of their audience.

F. Porikli observed that there is an imagination gap between the generation who grew up watching Star Wars episodes and earlier generations. People who have watched IMAX movies tended to imagine 3DTV as a miniature version of the movie theater in their homes. Younger generations are more open to the idea of a holographic tabletop display. In any event, people imagine that they will be able to move freely in the environment and still perceive the content in full 3D (which can lead to dissapointment if the viewer position needs to be restricted). Since conventional TV viewing is a passive activity, people do not usually have the expectation that they should be able to interact with the scene or have any effect on the program they are watching.

\subsection{Lay Persons' Expectations}

What do lay people expect from such a product? What features, function, and quality do they expect?

Today people take for granted high-quality 2D images and it would be unrealistic to expect them to put up with even moderately lower quality images in 3DTV. If the images are not clear, crisp, or if they are hard to look at in any way, it is unlikely that people will watch it. For a significant amount of TV content, 2D screens are already realistic enough, as M. Kautzner noted. Although in a technical sense one may think that $3 \mathrm{D}$ is more "realistic" than $2 \mathrm{D}$, that may be a fallacy. "Realisticness" is very psychological: a clear, crisp color image is very realistic to a lot of people watching TV or a film, whereas a 3D image which deviates even a little from this crispness and contrast may look terrible. Humans possibly do not really miss true 3D information, since they can deduce enough of it from the content. Human imagination is such that even if we see a reduced representation of reality, such as a black-and-white photo, a 2D image, or even a sketchy caricature, we can fill it in our minds and visualize its realistic counterpart. Black and white photos are quite realistic despite the loss of color information. Other than an arrow flying towards 
you or a monster jumping at you (contrived actions familiar from the old colored-glass 3D films), it is not clear exactly what information 3DTV is going to convey that will be important to viewers. Thus if the only thing 3DTV has to offer is the novelty factor, it will not be a mass market. The opposite argument could be that, by the same token, people did not really need color information either; black-and-white TV was just fine, but color TV still caught on. Nevertheless, the introduction of color did not entail much sacrifice of quality; G. Ziegler remarked that 3DTV will have a difficult time if it is of lower quality and this will be all the more true if it is difficult to watch or strains the eyes.

Some consumers expect to see the same kind of an aquarium as the contemporary TV is, but somehow conveying some sense of depth. Other consumers expect to be able to move around the display freely and be able to see the view from different angles. Another group of consumers totally lacks any vision of "true" 3D, and merely expect 3D graphics on a flat panel, as in current 3D computer games. And a significant group of consumers seems to have hardly any idea of what the term might imply. These observations imply that it may be important to educate potential consumers that the $3 \mathrm{D}$ we are talking about is something more than the $3 \mathrm{D}$ of a perspective drawing.

F. Porikli noted that while non-entertainment users of 3DTV may be willing to forego several comfort or convenience features that are not pertinent to the application, the expectations of household entertainment consumers may be higher. People do not like the idea of wearing goggles or markers or beacons, and they certainly do not like having limited viewing positions or low resolution. Consistency of $3 \mathrm{D}$ image quality with respect to viewer motion and position is another important factor. As for price, Porikli believed that any display product costing over 5000 USD is not likely to be widely accepted.

I. Rakkolainen argued that rather than trying to achieve a perfect $3 \mathrm{D}$ display, tricks and approximations must be used to obtain a reasonably priced and good-enough display for general use. Indeed, while R\&D group A may be focused on "true" or "real" holographic reconstruction, R\&D group B may get to market with a sloppy, pseudo-, quasi-, and a really not-deserving-the-name product which nevertheless satisfies these conditions. The question is, what aspects of 3DTV will be important and attractive to consumers and which will be irrelevant? Maybe true 3D parallax, and the ability to walk around which are hallmarks of true 3D may not matter; maybe people will be comfortable simply with more depth cues on a flat screen. J. Kim noted that in fact, in many cases 2D cues are sufficient for depth perception.

N. Stefanoski commented on the issue of whether more information is always desirable. In some cases, conveying the maximum amount of information may be desirable (perhaps for sports events, teleconferencing, virtual shops), but in other cases there will be not much consumer desire to choose the viewing perspective. In fact in some cases fixing the perspective may be desired 
for artistic reasons or genre convention (hiding the face of the murderer in a mystery film).

A. Boev noted the importance of studying consumer expectations. Although consumers are often "taught" what they need in the case of some products, for a novel and potentially expensive product it may be important to know what the buyers expect. The Nintendo Virtualboy was promoted as a "3D game system" which made people expect images floating in the air. When people realized it only works with glasses, almost everybody was heavily disappointed, and it was a failure.

A. Boev also emphasized the importance of two-way compatibility. 3DTV sets should be able to display $2 \mathrm{D}$ programs and $2 \mathrm{D}$ sets should be able to display a $2 \mathrm{D}$ version of $3 \mathrm{D}$ programs. This would be a general expectation based on the history of the transition to color. F. Porikli also emphasized that at the very least, any $3 \mathrm{DTV}$ should be back-compatible to $2 \mathrm{D}$ content.

D. Kaya-Mutlu noted that TV is here being conceived largely as a visual medium, as a conveyor of visual information, and the viewer's relation to TV is being conceived mainly as a matter of decoding/processing visual information, as a matter of visual perception. This is understandable if we assume that the major contribution of 3DTV is taken as the enhancement of images. She pointed out that this misses other important components of TV content, such as talk, and more importantly other social functions beyond being an information conveyor - such as providing background sound, serving as an accompaniment, or as a means to structure unstructured home time. These are all functions of the household TV set and whether they will transfer to 3DTV may be important determinants.

\subsection{Sources of Public Perceptions and Conceptions}

What past or present technologies or fictional sources have influenced people's conceptions of such a technology?

Some of the answers collected were:

- Colored (or polarized) stereo glasses.

- Three-dimensional IMAX movies and other theme park movies.

- Depiction of such technologies in science fiction movies and novels, such as Star Trek and Star Wars.

- Still holograms.

- 3DTV computer games or similar rendered objects on conventional TV.

- Virtual reality or augmented reality.

S. Fleck noted in particular that 3D theaters in Disneyland, Europa-Park, etc. and IMAX theaters might have had the greatest influence; 3D versions of Terminator 2 and The Muppet Show are popular examples. 


\subsection{Potential for Novelty Consumption}

Is there a novelty-oriented segment of the population willing to pay for expensive, relatively low-quality early consumer models?

R. Civanlar did not think that an early model of low quality regarding resolution, color, etc. would be acceptable; consumers are too accustomed to high resolution crisp images. However, low quality or restrictions on the 3D features may be acceptable since consumers have not yet developed high expectations in that regard yet.

Audiences might at first rush to watch the new 3DTV tabletop football games, but the novelty would quickly fade after a couple of times and people would probably return to the comfort of their 2D sets. M. Kunter made a similar comment about IMAX theaters, which remain a tourist attraction but have never become established as cultural institutions like common movie theaters.

G. Ziegler thought that there may be a subculture of science fiction enthusiasts who would gladly pay for initially expensive hardware - not so much for the content they would watch, but for the excitement of the experience they are familiar with from science fiction. He noted that 3D already has the status of a hobby with specialist suppliers such as www.stereo3d.com, which evaluates all kinds of exotic hardware from small and large companies. Purchasers of this equipment are not ordinary consumers but hobbyists who sometimes modify the hardware. Ultimately, however, this group is small and without large buying power.

Ziegler also noted that certain rich urban singles often have an interest in such gadgets; for them the design is of paramount importance, even more so than the features. G. Ger and Ö. Sandıkçı both thought that certain highincome customers might buy such a product for the sake of novelty if it were a status symbol; but they felt that such an outcome is socially divisive and not desirable.

I. Rakkolainen emphasized that it might make more sense to target the early models at businesses rather than the consumer of novelties; businesses, the military, and other special applications customers can pay significantly greater amounts and take greater risks. He pointed out that some rich consumers might buy expensive technology if it gave them something new, fun and useful. But he wondered if there are enough such consumers. The same seems to be the case for non-mainstream customers who are so attracted by the novelty that they are willing to put up with low quality. (P. Surman was of the opinion that such populations are more likely to be motivated by being the first to own a product, rather than being thrilled by the novelty factor.) Therefore, focusing on non-consumer markets seems to be strategically more advantageous. Ziegler also noted that major companies like to use novel yet expensive technologies at fairs for promotional purposes. F. Porikli supported Rakkolainen, noting that some people pay huge sums for expensive artwork and hobby cars, so there is obviously a market for everything, but how big is 
that market? Without convincing content support, Porikli thought it unlikely that expensive 3DTV products will ever reach any but the richest people. He also emphasized the importance of the non-household market: research labs, assisted surgery and diagnosis in medical settings, military applications, and video conferencing.

V. Skala believed that the hand game industry might be an engine for future development. A. Ö. Yöntem also believed that there will be a significant demand for game consoles with 3D displays, generating considerable revenue.

R. Ilieva pointed out that one option in introducing 3DTV to the masses would be an approach involving small changes to ordinary TV sets. H. M. Ozaktas noted that for instance, K. Iizuka of the University of Toronto has produced simple add-ons for cellular phones allowing them to transmit stereo images. Similar approaches may be technically possible for 3DTV, but it is not clear whether these would interest consumers.

I. Rakkolainen reported a quotation from Alan Jones in the newsletter 3rd Dimension (www.veritasetvisus.com), Jones suggested that a new level of technology must drop to 5-10 times the price of its predecessor to get users interested; when the price drops to only double it starts getting widespread acceptance from early adopters. The price must fall to about 1.5 times the predecessor's before it can become a truly mass product. In summary, Jones felt that the future for $3 \mathrm{D}$ displays is bright but they will not displace $2 \mathrm{D}$ because there will continue to be uses for which $3 \mathrm{D}$ is not necessary.

\subsection{The Future Acceptance of Three-dimensional Television}

Will commercial three-dimensional television replace two-dimensional television, or will it remain as a novelty limited to only a certain fraction of consumers?

Many people seem to think that 3DTV may replace common television in the future. However, this thinking may reflect nothing more than a simpleminded extrapolation of a linear conception of progress moving from radio to television to color to 3D.

It is important to understand the acceptance of new technologies in the context of competition between rival market forces. D. Kaya-Mutlu recounted that in the 1950s, when TV had become a serious alternative to cinema, the American movie industry introduced technological novelties to attract viewers back to the movie theatres. The first novelty was 3D movies but these did not have a longlasting impact on viewers. The special eyeglasses that were required were the main reason for audience resistance. This is why many present researchers consider it important to develop approaches which do not necessitate the wearing of such equipment. The next novelty was Cinerama, which created an illusion of three dimensionality without special eyeglasses. It garnered more public interest, but it too became a tourist attraction in a few places, 
probably because it required a different and complex exhibition environment. Finally, it was the wide-angle process, Cinemascope, introduced by Twentieth Century Fox in 1953 that had the most longlasting effect among these novelties. Cinemascope movies offerred a wider screen image with color and stereo sound, and therefore contrasted sharply with the small, black and white TV image. Cinemascope movies also augmented the impression of telepresence. HDTV, which combines a large, high quality image with good quality sound, is an extension of this concept into the private home. Kaya-Mutlu thought that although it seems to be nothing more than a high-resolution version of ordinary 2DTV, HDTV could be a rival to 3DTV in the home market.

G. Ger noted that the different phases of acceptance of a new technology must be carefully studied to avoid strategic mistakes. She gave several examples of failed technologies, such as 3D movies and the picture phone. The picture phone had been the subject of science fiction for a long time; the public was already familiar with the concept and was even anticipating it. It seemed like the logical next step; just as television followed radio, picture phones would follow ordinary phones. The engineers working on it probably thought that price was the only obstacle and that would surely drop in time. But it turned out that although people sometimes wanted to see the person they were talking to, more often they did not. Perhaps you are unshaven or without makeup during the weekend, or perhaps you do not want your body language to tell your boss that you are lying when you say you are too ill to come to work. Ger underlined that a technology is accepted if it fits well with the existing culture and needs of society. On the other hand, H. M. Ozaktas noted that the present acceptance and popularity of free internet-based video-telephony (most notably Skype) should make us rethink these explanations; this could provide a lot of new data regarding people's acceptance and the factors underlying it. There are some useful questions: how do people use Skype, when do they prefer no video, when do they prefer to remain silent or inaccessible, how do they combine voice and the accompanying chat features.

Ö. Sandıkçı noted that about eighty percent of new products fail, mostly because of a lack of understanding of consumers and their needs. She also gave the example of 3D movies, noting that it had the image of being weird and juvenile kid stuff, which probably guaranteed its failure. She warned that the association of 3DTV with 3D movies could hurt the success of 3DTV. She talked of the need to think about how the technology will fit into the people's lives. For instance, referring to the tabletop 3DTV scenario, she noted that in a typical living room layout, the TV is not in the middle of the room. Therefore either the technology may effect the way people furnish their living rooms, or if it asks for a major change from people, it may face resistance.

D. Kaya-Mutlu had already noted the fallacy of conceiving of TV merely as a visual medium, and of the viewer's relation to TV as merely a matter of decoding/processing visual information. Another important component of TV content is talk and many TV programs are talk-oriented. More importantly, 
ethnographic research on TV audiences (within a cultural studies framework) exploring the significance of TV in the everyday lives of families and housewives, has shown that TV has several social functions beyond being an information conveyor. This body of research has shown that the pleasures derived from TV content are not merely textual (which includes both the visual and the aural information). For example, James Lull, in his article "The Social Uses of Television" (1980) develops a typology of the uses of home TV, which are not directly related to the content of TV programs. Distinguishing between structural and relational uses of TV, Lull points to the use of TV as a background sound, as an accompaniment, and as a means by which family members, especially housewives, structure unstructured home time. Lull also discusses how TV regulates the relations between family members. It has also been shown that TV is watched in an unfocused manner, at the same time as conversation and other domestic activity. Another aspect of this unfocused watching is the growing practice of zapping among channels. Kaya-Mutlu said that Ö. Sandıkçı was very right in pointing to the need to talk about how the technology will fit into the lives of people. Since 3DTV will cater to an audience whose expectations and viewing habits/styles have been shaped by 2DTV content, its realistic images may not be enough to attract a wide audience. She thought that 3DTV assumes a focused/attentive viewer while some evidence shows that many viewers watch TV in a distracted manner (for example there are housewives who "watch" TV without even looking at the screen; they are maybe more appropriately referred to as "TV listeners" rather than TV viewers). At the least, one can argue that 3DTV's popularity may depend on a radical change in the viewing habits and styles of the majority of viewers.

M. Karamüftüoğlu suggested that in order to avoid failure, it is important to talk with sociologists, philosophers, cultural theorists, and media artists. The essential ingredient of a successful commercial design is to iterate the design through interaction with potential consumers. He also noted the possibility of consumer resistance to obtrusive gadgets.

One of the most important issues brought up in this context was that of content. A 3DTV set does not mean anything without $3 \mathrm{D}$ content. The content is the real product, the set is just the device needed to view it. What is to be sold is the content, not the set. For instance, a typical CD or DVD player costs much less than the cost of any reasonable CD or DVD collection. And for content to be produced there must be demand, which can come only from customers already owning 3DTV sets, creating a chicken-and-egg situation. Therefore, Y. Yardimcs speculated that even if there were a small group of customers attracted to novelty, and even if they could support the production of the sets, would they reach the threshold necessary to justify the production of content? On the other hand, Yardimci also cited research showing that purchases of high definition (HD) television sets was rising at a faster rate than those receiving HD programming. This was paradoxically setting the stage for a boom in content production and thus the solution of the 
chicken-and-egg problem. F. Porikli, on the other hand, noted that a lesson learned from HDTV acceptance was that without sufficient content, it is not realistic to expect people to make such an investment.

R. Civanlar thought that acceptance will probably depend on the type of content. People may be willing to pay extra for 3D sports viewing. As for entertainment, special movies that use 3D effects would have to be produced. He mentioned a Sony theater in New York City that frequently shows high quality $3 \mathrm{D}$ feature films and is usually full even though the tickets are not cheap. On the other hand, although this particular theater has been around for ten years or so, no new ones have opened. He believes that Sony produces special movies for this theater, probably not to make money but for reasons of prestige and promotion.

Another aspect of the content issue was brought forward by D. Kaya-Mutlu, who noted that each medium has its own esthetics. For example, Cinemascope promotes long shots instead of close-ups, whereas the small low-resolution TV screen promotes close-ups. That is partly why many major cinematic productions look crammed and are less pleasant to watch on TV. Kaya-Mutlu suggested that the growing popularity of HDTV is likely to prompt some changes in TV esthetics; these may also be valid for 3DTV.

I. Rakkolainen suggested that 3DTV technology could be used with many different kinds of content. Apart from broadcast TV, some of the content categories could include 3D games, virtual reality, and web content. He also noted that an interim appliance might be an ordinary TV most of the time but be switchable to $3 \mathrm{D}$, perhaps with less resolution, when there is a special broadcast.

According to G. Ziegler, in order for big media companies to produce the content that would drive consumer demand, what is desperately needed is a common standard for 3DTV productions (this would at first be for stereo 3DTV systems). Naturally, standardization requires a certain degree of maturity of a technology; he noted that some of the new stereo movies available may set a de facto standard until better standards are agreed upon.

F. Porikli believed that consumers will not stubbornly stick to 2DTV if the quality of 3DTV matches expectations. Even though transitions, such as to HDTV, are painful, people find it difficult to go back once they are accustomed to the higher quality content. Nevertheless, $2 \mathrm{D}$ displays will continue to be used in many applications due to their cost, their smaller size, and their robustness.

Another issue is whether 3DTV would ever become standard or whether only selected special programs would be 3D. This may also depend on the restrictions and requirements $3 \mathrm{D}$ shooting brings to stage and set, an issue which does not seem to be widely discussed. Transition from radio to TV brought tremendous changes, whereas transition from monochrome to color brought only minor ones. If the requirements coming from $3 \mathrm{D}$ shooting are excessive, it might not be worth the trouble and cost for programs where it 
does not have a special appeal, and it may be limited to specific program categories including sports and certain film genres.

J. Kim noted that since it was not yet clear what shape 3DTV will take, it was not easy to comment on public acceptance. 3DTV may evolve from stereoscopic 3DTV requiring special glasses, to multi-view autostereoscopic 3DTV, and finally to holographic 3DTV. Each type of 3DTV could engender a different response. For the first two types (which lay 3D functionality on top of existing $2 \mathrm{D}$ without replacing it) the primary determinant of acceptance will be how the added 3D video services fit users needs for specific content types. These first two types of 3DTV should be backward-compatible-users should be able to switch to $2 \mathrm{D}$ viewing without losing anything more than depth perception. They should also be able to handle 2D/3D hybrid content. 3DTV systems, especially early ones, might exhibit various distortions, which would induce psychological fatigue with extended viewing. J. Kim therefore predicted that only selected programs would be shown in 3D mode.

\subsection{Other Consumer Applications of Three-dimensional Television}

Other than being a three-dimensional extension of common TV and video, what other consumer applications of 3DTV can you think of? (games, hobbies, home movies, automotive, smart apartments, etc.)

I. Rakkolainen believes that it is useful to distinguish between passive applications such as TV and video, navigation aids in cars, and interactive applications such as games. He also noted that the success of different applications will depend on the size and format of the displays that can be produced. He said that games and entertainment applications hold a lot of promise, because they can be adapted to many different display types. Indeed, many consider games an important potential application of 3DTV. A. Ö. Yöntem believed that a 3D game console designed to be connected to a 3DTV set would be very attractive to consumers. F. Porikli also agreed that since current TV displays support games, hobbies, entertainment content etc., it was likely that 3D displays would also do so. While many understand these to be more realistic and immersive versions of existing computer games, G. Ziegler suggested several less immediate examples. He first noted the success of the IToy, a camera-based device with simple 2D motion tracking; this is used for games but it has other applications - it can be a personal training assistant that supervises your daily exercises. If optical 3D motion capture works reliably, such systems could easily be extended in exciting ways. He also noted the DDR (Dance Dance Revolution), the Japanese dancing game, as an example of consumer interest in such devices and activity games.

Video conferencing is another potential application area. While many kinds of systems are already available for remote multi-party conferencing, they have still not replaced face-to-face meetings. Precisely what important features of 
the face-to-face interaction are lost, and whether they can be provided by 3DTV, remain interesting questions. F. Porikli commented that just as Skype users find it difficult to go back making traditional phone calls, people who experience $3 \mathrm{D}$ teleconferencing may not be willing to go back to conventional teleconferencing.

P. Surman noted that the display systems being developed have the capacity to present different images to different viewers and this could be exploited for certain purposes, such as for targeted advertising where a viewer is identified and an image intended specifically for that viewer is not seen by anyone else. This could work for more than one viewer. Such technology could also be used to block undesirable scenes from young viewers. A TV set which can display two channels simultaneously to viewers sitting in different spots has already been introduced, and marketed as a solution to family conflicts about which station to watch.

G. Ziegler noted potential applications mixing the concepts of 3DTV and augmented reality, where multi-camera recordings are projected into augmented reality environments. He also noted that there were several possibilities, such as the use of a webcam or a head-mounted display for "mixed reality" 3DTV viewing. Other applications include a virtual tourist guide and a virtual apartment walk-through.

J. Kim mentioned TV home shopping; people would like to see the goods as if they were in a store and would appreciate the added 3D depth perception and the ability to look around objects. Only the goods for sale need to be shown in $3 \mathrm{D}$, against a $2 \mathrm{D}$ background that includes the host and any other information. This mode of 2D/3D hybrid presentation could also be used for other programs such as news, documentary etc.

Applications of 3D displays to mobile phones was suggested by A. Ö. Yöntem, who argued that consumers would like to see a miniature of the person they are talking to. In this context, he also proposed the intriguing idea that 3D displays may form the basis of 3D "touch screens," although there are many questions about how to detect the operator's finger positions and purposeful motions.

\subsection{Non-consumer Markets for Three-dimensional Television}

What major markets other than the mass consumer market may arise? In other words, what could be the greatest non-consumer applications of 3DTV, in areas such as medicine, industry, scientific and professional applications, etc. Do these constitute a sizable market?

We have already noted I. Rakkolainen's position that such technologies should initially target business customers in high-cost professional areas like medical, military, and industrial visualization, followed by medium-cost applications like marketing and advertising. P. Surman also noted that such 
applications constitute a sizeable market and that these niche markets can justify a more expensive product. This could be useful for the development of a commercial TV product as it could take ten years to develop an affordable TV display, but less time to produce a more expensive one. The niche markets would drive the development. Likewise M. Özkan believed that due to the cost of initial products, professional areas such as military training, industrial design, and medicine were likely early application areas. R. Ilieva, along with others, believes that there is considerable potential in the medicine, education, and science markets.

While most agreed that industrial markets could tolerate higher prices, it was not clear that they would tolerate lower quality. T. Erdem noted that industrial applications may require even higher quality than consumer 3DTV applications. The consensus was that it depended on the application and could go either way.

G. Ziegler noted that 3DTV research may have many spill-over effects, in areas such as image analysis and data compression. This could lead to advances in areas such as real-time camera calibration, industry-level multicamera synchronization, real-time stereo reconstruction, and motion tracking. For instance, classic marker-based motion tracking (also used in the movie industry) might become obsolete with the advent of more advanced markerless trackers that stem from the problem of $3 \mathrm{D}$ data generation for free viewpoint video (FVV) rendering. Other applications might include remote damage repair, space missions, spying and inspection operations, remote surgery, minimally-invasive surgery, and regrettably military operations such as remote-controlled armed robots. F. Porikli added remote piloting and virtual war-fields to the potential list of military applications.

An interesting point was made regarding professional applications. In some professional areas, the existing values, norms, vested interests, or skill investments of practitioners may result in resistance to the technology. While most physicians are used to adapting to sophisticated new equipment, years of clinical training and experience with 2D images may make them resistant or uncomfortable working with 3D images. Also, their expectations of quality may be quite different than general consumers. As with many technologies, the issues of de-skilling and retraining arise. Many professionals learn over years of experience to "feel" the objects they work with, and when the technology changes, they cannot "feel" them any longer and feel almost disabled. As a specific example, S. Fleck noted that they have been doing research in the field of virtual endoscopy for years, and that they have asked surgeons about their opinion of 3D visualization. While about two-thirds said that they would appreciate such capabilities, it was important for them to be able to use any such technology in a hassle-free way with very low latency and high spatial resolution. They also insisted on maintaining the option of being able to fall back on the $2 \mathrm{D}$ visualization they were used to; they wanted to be sure that whatever extras the new technology might bring, they would not lose anything they were accustomed to. This is quite understandable given the 
critical nature of their work. K. Ward thought that as a doctor herself, she observed great fear among the medical profession that new technology may not be as safe; anything that doctors do not have experience with feels less safe to them and they hesitate to risk a bad outcome for their patients.

M. Özkan noted another potential reason for resistance from the medical establishment, which in theory should greatly benefit from $3 \mathrm{D}$ visualization in both training and practice. He underlined the resistance to even lossless digital image compression techniques for fear of costly malpractice lawsuits, and so was pessimistic regarding the adoption of $3 \mathrm{D}$ techniques in practice, but thought they may be more acceptable in training, especially remote training. J. Kim reported on trials in Korea applying different kinds of information technology to medicine. He referred to two big issues: broadband network connection among remotely located hospitals and doctors for collaborative operation and treatment, and exploitation of $3 \mathrm{D}$ visualization technologies for education and real practice. Accurate 3D models of human organs, bones, and their $3 \mathrm{D}$ visualization would be very time- and cost-efficient in educating medical students. Doctor-to-doctor connections for collaborative operations is considered even more necessary and useful than doctor-patient connections for remote diagnosis and treatment. Kim believes the medical field will surely be one of the major beneficiaries of 3DTV.

H. M. Ozaktas noted that many examples of resistance to new technology is available in consumer applications as well; a new car design with different positions of the brakes, accelerator, and gearstick, would not easily be accepted even if tests showed it was safer and gave the driver better control. Likewise, despite its clear inferiority, the QWERTY keyboard is still standard and very few people attempt to learn one of the available ergonomic keyboard layouts.

A number of participants, including C. Türün and V. Skala emphasized the education market. Skala gave several examples from three-dimensional geometry where students had difficulty visualizing shapes and concepts; 3DTV may help them improve these skills. Indeed, the traditional book culture as well as the more recent visual culture are both heavily invested in 2D habits of thinking. H. M. Ozaktas agreed that perception of 3D objects may be improved with the use of 3D imaging in education, but argued that the applications to education should not be limited to this, suggesting that we should be able to, for instance, show simulations of a vortex in fluid mechanics or the propagation of a wave in electromagnetics. However, even very low-tech 2D animations which can add a lot to understanding are not often used in educational settings, despite their availability. Ozaktas gave the example of simple animations or simulations of electromagnetic waves and how useful they could be, but noted that most electromagnetics courses do not include such simulations. He concluded that customary habits and possibly organizational obstacles may come before technical obstacles in such cases. 


\section{Part III: Social Impact and Other Social Aspects}

\subsection{Impact Areas of Three-dimensional Television}

Will the greatest impact of 3DTV be in the form of consumer broadcasting and video (that is, the three-dimensional version of current $T V$ and video), or will the greatest impact be in other areas such as medicine, industry, scientific applications, etc?

P. Surman believed that the greatest impact will be in the form of consumer broadcasting and video, since this will potentially be the most widespread application. I. Rakkolainen agreed that this may be the case in the long run; in the meantime the greatest impact will be in special experiences created by the entertainment industry with high-end equipment. He noted that there are already very low-cost head-mounted displays for PCs and game consoles, but they have not yet sold well, although they could become popular within 5-10 years. V. Skala also agreed that in the long run consumer 3DTV will have the greatest impact but meanwhile other professional areas will have a larger impact.

\subsection{Social Impact of Three-dimensional Television}

Television is currently understood as being a social ill. Its negative effects, including those on children, have been widely documented and are considered to far outweigh its positive aspects. In this light, what will be the effect of 3DTV technology? Will it further such social ills? Will it have little effect? Can it offer anything to reduce these ills?

There is a vast literature regarding the negative effects of ordinary television on children. The negative effects mentioned include the conveying of a distorted picture of real life, excessive exposure to violence, obesity due to replacement of active play, unsociability due to replacement of social encounters, and negative developmental effects due to replacement of developmentally beneficial activities. In the early years, additional negative effects include negative influences on early brain development as a result of the replacement of real-person stimuli, and exposure to fast-paced imagery which affects the wiring of the brain, potentially leading to hyperactivity and attention deficit problems.

S. Sainov noted that during their holographic exhibitions, children $2-5$ years old and older people with minor mental deteriorations were very much impressed by 3D images; this suggests that the psychological impact of 3D images on TV screens should be taken into account. P. Surman noted that children are fascinated by 3D, suggesting this may be due to their greater ability to fuse stereo images.

R. Ilieva commented that although TV is a social ill, it has also had important positive aspects; it has brought knowledge of the world to low income 
people who cannot travel and do not have access to other sources of information. 3D technology can have a positive impact on science education but it is not clear how much 3D can add to the general information dissemination function of TV. P. Surman thinks it will have little effect, since there was no noticeable difference when color took over from monochrome. F. Porikli however thinks that both positive and negative impacts would be enhanced since 3DTV has the potential to become a more convincing and effective medium than conventional TV.

A. Boev noted that a social ill is something that hinders the basic functions of society, such as socializing; by this definition, TV is a social ill, but Skype is not; playing computer games is a social ill, but writing in web-forums is not. He said that even reading too much could be a social ill. He argued that maybe the main feature which makes TV a social ill is its lack of interactivity. If TV was truly interactive (and went beyond just calling the TV host to answer questions), it would not be such a social ill. M. Karamüftüoğlu noted that this view can be criticized; for instance, some would argue that certain forms of Internet communication such as chats are poor substitutes for face-to-face human communication, that they distance people from their immediate family and friends, and actually have an antisocial effect. Karamüftüoğlu believes that $3 \mathrm{DTV}$ can be less of a social ill than present-day television only if it can be made to and is used to convey more human knowledge. This should involve bodily, embodied tactile interaction and immersion with affectivity and subjectivity.

G. Ziegler pointed out that there has been a radical transformation in the social isolation associated with playing computer games; many games are now networked and played interactively, sometimes in large role-playing communities. While these games may separate you from your local community, they make you a member of other communities. Potentially such games may still be considered a social ill, given that they may isolate people from family, school, and work contacts. On the other hand, being able to network with others who share common interests and not being limited to people in ones immediate social environment, appear to be beneficial.

I. Rakkolainen pointed out that it is not so easy to claim that TV (or networked games, Skype, books) is a social ill for all. Some people get seriously addicted to TV, watching it 10 hours a day, while others may get addicted to excessive gardening, football, music playing, drawing, virtual reality, drugs, sex, and so forth in an attempt to escape real life. He argued that if anybody gets addicted to these things, the reason is usually not in the particular thing or technology, but somewhere deeper in their personality or history. Nevertheless, he agreed that new lucrative technologies can make the old means of escape even more effective. Will 3D technologies have such an effect? Some people were already addicted to computer games in the $1980 \mathrm{~s}$, but the advent of superior graphics, 3D displays and virtual reality will make it much easier for the masses to get immersed. Interactive technologies are more immersive, as they 
require continuous attention. In the end, the implications of A. Boev's thesis that interactivity might improve the status of TV, remained an open issue.

M. Özkan and J. Kim agreed with Rakkolainen that the technology is not intrinsically good or bad, but it is how it is used that makes it good or bad; lack of social interaction seems to be a growing problem in the developed world and Özkan was not sure that it was fair to blame TV for it. He noted that the erosion of extended families, the disappearance of communal structures and living conditions such as old style neighborhood interactions and neighborhood shops have all contributed to social isolation. V. Skala agreed with him that the major negative aspects of TV were based on its use as a tool to boost consumption and to indoctrinate people; he added that TV programmers do not try to produce value but just use sophisticated psychological techniques to keep people passively watching TV. On the other hand, M. Özkan continued, TV could potentially be used as an effective and economical education tool if public-interest broadcasting could be more widespread. Given the current trends, the move to 3DTV may increase its power and negative effects, a point also agreed to by Kim.

N. Stefanoski suggested that the spectrum of different applications of 3DTV technology will be much wider than traditional television, with potential applications in the areas of medicine (telesurgery, surgery training, surgery assistance), industry (CAD), and military (training and simulation in virtual environments). Immersive $3 \mathrm{D}$ environments could be created to improve the social environment of elderly and handicapped people, helping them to have more realistic-looking visual contact with other people and interact with them. Thus, in judging the overall effects of the technology, we should not focus only on consumer 3DTV, but also consider the array of potential non-consumer applications which may have a considerable benefit to society.

D. Kaya-Mutlu noted that the question under discussion frames TV around the "effects" model of mass communication. However, this model/ theory of "strong effects" or "uniform influences" was challenged in the 1930s, $40 \mathrm{~s}$, and 50 s (i.e., the uses and gratifications approach to media consumption). It was shown that the media do not affect everybody uniformly; individual psychological differences, social categories (e.g., age, sex, income, education), and social relationships (e.g., family, friends, acquaintances) affect people's perception and interpretation of media content. In the 1980s, culturalist studies of audiences showed that consumers are not passive recipients of encoded meanings and identities in the media. These studies redefined media consumption as a site of struggle. For example, Stuart Hall has argued that representations of violence on TV are not violence per se but discourses/messages about violence. Hall, David Morley and other audience researchers showed that, depending on their social, cultural, and discursive dispositions, viewers are able to negotiate and even resist media messages. 


\subsection{Comparison to the Move to Color}

Will the effects of moving to three dimensions be similar to the effects of moving to color?

A. Boev believes that merely adding another dimension (color, depth, even haptics or olfaction) to TV is not going to greatly affect the social impact of such a medium. On the other hand, P. Surman believes that once viewers have become accustomed to watching 3D images, 2D images will appear dull and lifeless. R. Ilieva also believes that the move to $3 \mathrm{D}$ will be more important than the move to color.

C. Türün noted that the present state of $3 \mathrm{D}$ displays are not yet of sufficient quality to allow us to imagine what it might be to experience 3DTV where the images are almost impossible to distinguish from the real thing. If such a high quality image is hanging in the air and is not physically attached to a screen, people might have an experience which is difficult for us to imagine now. In this case, the move to $3 \mathrm{D}$ would be much more significant than the move to color and may be comparable to the difference between a still photograph and a moving picture. G. Ziegler agreed with Türün, underlining that present display technologies which are not quite "true" 3D are not significantly different from ordinary television. He also noted that a more challenging target than the move to color might be the move to moving pictures: could we ever create the awe that the first moving pictures generated in the audience? M. Özkan also agreed that only transition to a "real" 3DTV system could be much more important than the transition to color.

However, many believe that in any event, interactive TV or immersive TV is almost certain to have a much larger impact than 3DTV. In other words, the addition of the third dimension may have less impact than the possibility of interaction or immersion. G. Ziegler noted that even very convincing 3D displays, if in relatively confined spaces and viewing conditions, will be far from creating the immersive experience of even ordinary cinema. This seems to indicate that simply the size of the display and the architecture can have more of an immersive effect than the perception of three dimensions. Therefore, G. Ziegler suggested that it would be worth investigating large-scale 3D display options, even if they were not true 3D or they offered only a limited amount of depth perception; these might create a much more breathtaking experience than true $3 \mathrm{D}$ systems in confined or restricted viewing conditions.

\subsection{Economic Advantages of Leadership}

If Europe, the Far East, North America, or some other block becomes the first to establish standards and offer viable 3DTV technologies, especially to the home consumer market, what economic advantages may be expected? 
P. Surman noted that for Europe, the value added could be from licensing and from the fact that there are no overwhelming barriers to displays being manufactured in Europe. R. Ilieva believed that if Europe became the first player to establish the standards and offer viable 3DTV technologies, especially to the consumer market, the economic advantages would compare to that for CDs and DVDs.

V. Skala didn't think that Europe would be the main player. He also expressed pessimism regarding standards: they would take a long time to develop and meanwhile the market would have already moved on. He thought that while there will be similar principles of coding, there will also be many variations (like NTSC, PAL, SECAM). He guessed that major Far Eastern countries may once again take the lead. G. Ziegler was also skeptical of Europe's capacity to provide leadership, based on observation of earlier technologies such as HDTV and GPS. Nevertheless, he argued that if the EU could set forth certain common standards, then it might give media producers and hardware manufacturers a huge home market for the fruits of their latest research; and if the rest of the world finds the new medium desirable, then these companies will have an advantage. He also underlined that it is the media standardization and the following media content which generate the revenue, not the hardware. M. Özkan also agreed; the size of the EU market makes it viable for consumer electronics companies to achieve economies of scale in producing a new EU-standard 3DTV. However, he also noted that the added value is not in the hardware but in the content. For traditional broadcast TV, the commercial model was simple: consumers pay for the equipment (so manufacturers target the end-consumer-branding and marketing is important) and advertisement revenue pays for the content. With the move to Digital TV, it has been mostly the service provider who pays for the equipment and recoups this cost from the monthly service charge to the end-consumer. On most equipment, the manufacturers brand is either invisible or is clearly dominated by the brand of the service provider. A parallel business model has been in the works for game platforms. In such "service provider" subsidized equipment models, low-cost manufacturers have a clear advantage, and famous brands end up having a cost disadvantage because of the brand marketing costs (among other things) they incur. However, establishing a standard obviously creates a great advantage for those companies who own the intellectual property and patents. Hence although "manufacturing" of the equipment might be done by non-European companies (or even European companies doing it offshore), if intellectual property is developed early on and included in the standards, that can establish a clear and lasting advantage.

C. Türün thought that with the current rate of development, no place is any more advanced than any other. But if something radical were to be achieved by a European company, such as an application much more extraordinary than mundane TV or film content, we would be able to talk about real economic advantages. 


\subsection{Comparison with Other High-impact Technologies}

How large might the impact of consumer 3DTV technology be, compared to other related established consumer technologies such as audio and video, cellular communications, etc.

P. Surman noted that the impact of 3DTV technology is likely to be high due to the high proportion of time people devote to watching TV. Also, viewing patterns are likely to change in the future; the TV set, the most familiar and easy-to-operate device in the home, will evolve into a media access gateway serving the information society.

In contrast, A. Smolic said that when he imagines the world before TV, he believes that the move to 3D would be nowhere nearly as important as the introduction of TV itself. 3DTV should be considered more as another step in the development of TV, rather than as a revolutionary new technology. Moreover, he predicted that 3DTV would not spread far quickly and broadly, but rather would develop from niches; perhaps it would never completely replace $2 \mathrm{DTV}$.

G. Ziegler approached this issue somewhat differently. Rather than thinking of end-to-end consumer TV, he looked at acquisition, compact representation, rendering, and display technologies separately. With regard to acquisition for example, he noted that being able to acquire 3D surfaces of yourself or your surroundings could be of great interest for immersive online games, where you could quickly create a 3D avatar of yourself. If $3 \mathrm{D}$ tracking can be made good enough, it will open up exciting new possibilities for game entertainment and would likely be popular. As for compact representation, being able to compress $3 \mathrm{D}$ video so that you can store it on a DVD could be of interest for documentaries, but not for feature films, since they may remain a rather passive experience with $3 \mathrm{D}$ being merely an add-on (as in IMAX 3D theaters). However, in video conferencing, 3D would probably increase the feeling of telepresence, and might be successful. Free viewpoint rendering is probably mostly of interest for documentaries or plays; this is a radical change in filmmaking since the director's control over point-of-view is lost. For this reason, free viewpoint movies may not appear soon. But, in video conferencing it would be very desirable to change viewpoints. In summary, many kinds of 3D displays could have market potential provided they did not cause eye strain, and provided there was a standardized media format (which did not soon become obsolete), and interesting high-quality media content.

S. Fleck noted that apart from TV programming in the conventional sense, many other forms of content for consumer 3DTV may emerge. The example he used was Google Earth; he noted some attempts to produce some basic anaglyphic (involving red and blue glasses) stereoscopic screenshots so it would be possible to experience "3D" in Google Earth. 


\subsection{Social Impact of Non-consumer Applications}

Which non-consumer applications of 3DTV-including such areas as medicine, industry, the environment, the military-may have significant beneficial or harmful impacts on society (Example: telesurgery.) What could be the extent and nature of these impacts?

M. Özkan noted the steady increase of non-conventional training methods in the military. The U.S. department of defense is specifically supporting software companies to develop game based e-learning and training systems. Such systems allow the military to train their personnel for very different environments and situations, without risking lives. These users would likely welcome a realistic 3DTV system. Even higher-cost early implementations of 3DTV may find use in military training systems. (Such systems can obviously be used for more humane applications, such as disaster readiness, first-aid, and humanitarian aid applications; unfortunately funds for such applications are much more limited.) G. Ziegler also noted implications for the battlefield such as more realistic virtual training environments. He was concerned that $3 \mathrm{D}$ visualization might make war become even more of a "computer game" to commanders and thus distance and alienate them from the resulting human suffering.

Özkan also noted that industrial designers, specifically designers and manufacturers of $3 \mathrm{D}$ parts and systems have always been very aggressive users of any 3D software and hardware that shortens production times. However, these applications might require very high resolution, thus creating the need for higher quality industrial grade 3DTV.

I. Rakkolainen observed that the trend in modern society to become very visual will be enhanced by any future 3D technologies; undesirable uses will be enhanced along with the desirable ones - violent video games will become even more realistic.

G. Ziegler believes that telesurgery will have a huge impact. Young students have no problem looking at a screen while using surgical instruments in simulated surgery. Apparently, kids who have grown up with computer games will be quite ready to adapt to augmented reality, making such applications a reality. Another example he gave was in the area of aircraft maintenance. Paper airplane repair manuals could run up to many kilograms of weight and are very hard to handle during maintenance because their pages easily become soiled. Augmented reality displays could provide the page content, but 3DTV technology could do even more by superimposing actual objects from the manual, such as screws, onto the field of view, and thus make it easier for the maintainers to do their job.

Video-conferencing is one area where the quality of face-to-face meetings can still not be recreated. If 3DTV could make video-conferencing more satisfactory, Ziegler pointed out it could reduce business travel considerably with enormous time and cost savings. H. M. Ozaktas added that this may be an incentive for companies to invest in rather expensive preliminary 3DTV models. 


\subsection{Implications to Perception of Reality}

Referring to the tabletop football scenario, M. Karamüftüoğlu noted that the first thing that comes to mind when $3 \mathrm{DTV}$ is mentioned is a telepresence type of experience. 3DTV is usually described as being realistic in the sense that it captures all the information and not just a $2 \mathrm{D}$ projection of it. However the perspective in the artist's depiction of tabletop football was not the natural perspective of a person in the stadium or on the field; it was rather a bird'seye view. The distance between the human observer and the players is very short and the scale is very different.

M. Karamüftüoğlu made two further points regarding realism. First, the greater the amount of information conveyed, and the more absolute the information becomes, the less there is for viewers to do and they will be pushed into a more and more passive role; this is already a criticism of conventional TV. Only the final dimension of realism - interactivity - could possibly reverse this. Indeed, the $3 \mathrm{DTV}$ image is absolute and real only in the passive sense; realism in a broader sense must also include interactivity. The main distinguishing feature between real and reproduction is not $2 \mathrm{D}$ versus $3 \mathrm{D}$, not black-and-white versus color, but the possibility of interaction with the actual scene as opposed to isolation from it. He explained that nothing short of tactile, bodily interaction with the objects would bring back realism.

TV content can be divided into two broad categories: factual programs and fictional programs, noted D. Kaya-Mutlu. Realism, especially in the case of fictional programs, should not be construed solely in the sense of realism of the images. One also needs to consider "emotional realism" (the recognition of the real on a connotative level). For example the relevancy of the characters and events in a film to the everyday lives of viewers may contribute much more to the impression of reality than the characters' realistic appearance; it is not the appearance of the characters which make them realistic but rather their actions, their relation to events and to other characters in the film. The contribution of 3DTV to increasing realism should be evaluated within this broader understanding of realism. Nevertheless, Kaya-Mutlu suggested that it is interesting to think about the contributions of $3 \mathrm{D}$ images to the production or enhancement of emotional realism itself.

Another very interesting topic was brought up by G. Ziegler, and elaborated in a response by $\mathrm{H}$. M. Ozaktas. Ziegler talked about the implications of mixing real and animated characters, especially animated characters with real skin; this produces an erosion of trust in video images. Animated images are obviously not to be trusted, and everyone knows that still photographic images can be altered by "photomontage." However, realistic video images are still trusted because people know they must be real shots of real people; people "know" that such images cannot be fabricated. But as everything becomes fully manipulable, we are entering an era where no media content can be taken to constitute "evidence"; you can believe it to the extent that you trust the source, but there is no true first-hand witnessing-at-a-distance any 
longer since virtually most forms of transmitted data will be manipulable, even the most "realistic" ones of today. And people will know it.

I. Rakkolainen joined this discussion by suggesting that with the digitalization of all forms of media, it would be possible to create immersive and interactive 3D experiences; eventually synthetic 3D objects would be indistinguishable from real objects captured with a camera. H. M. Ozaktas found this to be a strong statement, with interesting implications. Ozaktas recalled the famous work "The Work of Art in the Age of Mechanical Reproduction" by Frankfurt School author Walter Benjamin, who wrote in the first half of the 20th century. With "recordings" on media no longer just representing, but becoming indistinguishable from true things, art and esthetic theorists will have a lot to theorize on. Ozaktas surmised that maybe one can write about "The Work of Art in the Age of Indistinguishable Reproduction."

Ziegler thought that the ability to use digitized characters in computer games and maybe even films will make it possible to create or simulate nonexisting or existing digital actors. This could drastically reduce film production costs and also encourage pirate productions, with a host of interesting and complex implications. Ozaktas noted that the ability to pirate an actor digitally would open up copyright and ownership issues much more complex than the current pirate copying and distribution of copyrighted material. Also, the "star-making" industry will be transformed. A star may be a real person, but it will no longer be necessary for him or her to actually act, or even be able to act; he or she will merely be the model for the digital representation used in the films. In some cases, the star will not correspond to any real person, and will be nothing more than an item of design in a studio.

Ziegler also brought up the possibility of "reviving" human actors or personalities who are no longer alive through skeletal animation with realistic skin. The traditional capital of an actor was his/her actual physical presence, but now it would be merely his or her image, a commodity that can be sold and hired, even after the actor is dead. Celebrities like football stars or top models can be in Tokyo this morning in a televised fashion show and in Rio tomorrow for a talk show. Indeed, since most of us have never seen these people in the flesh, it is conceivable that totally imaginary personalities could be synthesized for public consumption. People may or may not know that these people do not actually exist, but perhaps it will be commonly accepted that they may not exist, just as we do not mind watching fictional films, knowing the events are not true.

$\mathrm{K}$. Köse noted that in certain circumstances, the availability of very convincing images could open the door to "persona piracy," the ability to convince others that you are someone else.

Ziegler also asked whether such media would offer alternative means of escape, involving addiction, and concluded yes, but since there are already enough paths to escape, this will not have a substantial impact. Mastery of even the earliest computer or video games demonstrated the potential for addiction and escapism. Realism may increase this considerably. But will highly 
realistic and interactive media allow qualitatively new levels of escapism from the real world? Presently, many substitutes for the real world exist, including imitation eggs and sugar, but in the social realm, substitutes are usually poor, nowhere near the real thing. If this changes, more people may prefer the more controllable, lower-risk nature of artificial experiences, leading to a society of isolated individuals simulating human experiences with quite genuine sensory accuracy. Although sensorily and therefore cognitively equivalent, these experiences will not be socially equivalent and will have an effect on how society operates. Unless, of course, computers are programmed to synchronize and coordinate the virtual experiences of individuals so that the resulting experiences and actions are in effect comparable to present social interactions and their consequences. For example, person A is hooked up to a simulator virtually experiencing an interaction with person $\mathrm{B}$, and person $\mathrm{B}$ is likewise apparently interacting with $\mathrm{A}$, and the two computers are linked such that their interactions do actually simulate actual interaction between them. In that case, the distinction between simulation and true interaction disappears; such simulation is effectively a form of remote communication and interaction.

Ziegler also argued that children who experience such media concepts will not be impressed by conventional fairy tales and lose interest in them. Generalizing to other aspects of human culture, it may be argued that poetry, novels, music, even traditional cinema images may no longer be able to capture the interest of audiences. The erosion of interest in poetry and the theater in the 20th century may support this, but the continued interest in at least some forms of printed content and plastic arts are counterexamples.

M. Karamüftüoğlu compared the 3DTV tabletop football scenario with the painting Anatomy Lesson by Rembrandt. He noted how the way the professor held his hand conveyed knowledge about human anatomy. He contrasted this embodied tactile/haptic human knowledge with the disembodied absolute/objective machinic knowledge of the tabletop football scenario. Indeed, engineers attempt to produce more "realistic" images by going from black-andwhite to color, from $2 \mathrm{D}$ to $3 \mathrm{D}$, and so forth, but they often seem to be moving towards such objective, physical/machinic knowledge, at the expense of more human knowledge. In the physicist's objective understanding of color, each wavelength corresponds to a different color, whereas human understanding of color is based on the three primaries, which is rooted in human physiology. Objective knowledge is independent from the human observer; thus "true 3D" aims to fully reconstruct a light field as exactly as possible and thus preserve as much objective information as possible. An artists rough sketch or caricature, on the other hand, may do very poorly in terms of any objective measure of fidelity and information preservation, but may carry a very high degree of information about human nature, even including such intangible things as psychological states.

These discussions are not necessarily restricted to 3DTV, but applicable to any technology such as 3DTV, which increases the accuracy and realism of remote experiences. For example, odor-recording technologies allowing the 
recording and playback of smells are being developed. These devices analyze odors, and then reproduce them by combining an array of non-toxic chemicals.

\subsection{Interactivity}

Although interactivity is not a defining characteristic of 3DTV, it comes up constantly in relation to 3DTV. Interactivity is a very important trend in all TV-type media, but it is possibly even more crucial for, perhaps even inseparable from, 3DTV. Interactive "TV" is almost certain to have a greater impact, good and bad, than 3DTV, regardless of the number of units sold.

A. Boev discussed different kinds of entertainment or "media" and their differing degrees of interactivity. Live events such as theater and sports events, offer a degree of interactivity with the opportunity to throw eggs, shout, sing, and engage in hooliganism. Historically, in some forms of staging the audience was allowed to shout, argue, or even decide on the course of action (gladiators). Nevertheless, with the exception of certain experimental theaters, being among the audience is generally a safe place to be, allowing one to be close to the action without risking too much. Different degrees of interactivity are sought by different audiences in different contexts and ignoring this fact might lead to the rejection of a product. There might be a few forms of interactivity - such as the choice of point of view - which are special to 3DTV.

In the same context, D. Kaya-Mutlu noted that interactivity necessitates an active viewer. However, in her view, the popularity of TV is based on its being a passive medium. Interactivity may not be much desired in a medium which is so associated with leisure.

\section{Part IV: Gender Related Issues}

\subsection{Effect on Gender Inequality and Gender Bias}

Can you think of any aspect or application of 3DTV that will increase or decrease gender inequality or bias in the social and cultural sense?

Most participants who expressed an opinion on this issue did not believe there will be any major effect, apart from what is discussed in the following section. However, it is important to underline that most of the discussion participants - as well as most developers of 3DTV technology - are male. G. Ziegler pointed out that it is worth looking into such biases in the areas of computer games, general computer usage, and usage of other "high-tech" consumer gadgets (although the insights gained may not be specific to 3DTV). He suggested that women would become more interested in using computers when the available applications and games have a considerable component of social interaction. Likewise, if 3DTV becomes a tool of social interaction, more women will become interested in it. 
Can you think of any applications of 3DTV that will benefit or harm men or women to a greater degree? (Example: Medical application treating a disease more common in men or women.)

Not too many examples were put forward, with the exception of the application areas suggested in the following section. Of these, the effects of pornography are largely perceived as negative, and more so for women. On the other hand, it has been argued that training and education applications may benefit women to a greater degree. Whether entertainment applications which selectively target men or women can be said to "benefit" them is open to debate. It was commented that application of 3DTV to shopping may also be viewed as being potentially exploitative.

\subsection{Gender-differentiated Targeting of Consumers}

D. Kaya-Mutlu noted that ethnographic audience studies have shown that TV viewing is a gender differentiated activity not only in program preferences but also in viewing styles. Researchers have found that while men prefer such programs as news, current affairs, documentary, and adventure films, women prefer quiz shows, serials, soap operas, and fantasy movies (mostly talk oriented TV genres for which 3D may not be necessary). But the home is a site of power relations and when there is a clash of tastes, masculine preferences prevail. Researchers have also identified some differences between the viewing styles of men and women; while men watch TV in a focused and attentive manner, women watch it in a distractive manner (i.e., together with at least one another domestic activity like ironing or feeding the children). Kaya-Mutlu concluded that these gendered program preferences and viewing habits imply that 3DTV, which seems to favor visual information and encourages focused/attentive viewing, may be more responsive to male demands and tastes; this may encourage producers to reserve 3D for prime-time programs since most daytime programs are addressed to children and housewives.

Can you think of any applications of 3DTV that will target either men or women as primary consumers? (Example: Broadcasting of male-dominated sports events.)

Three applications have been noted that may target men as primary consumers: male-dominated sports events, games applications, and pornography. It was suggested that such applications may help 3DTV by building initial niche markets. Y. Yardımcı and A. Smolic noted that in particular, 3D pornography may become a popular industry, but many participants expressed that they would be uncomfortable from an ethical perspective in building 3DTV's success on such grounds. If 3DTV does become a new tool in disseminating this type of content, the result will be to increase the negative and exploitative impact of such content on society. It was also commented that in any event, 
it cannot be taken for granted that $3 \mathrm{D}$ will make this type of content more attractive for its consumers.

A number of shopping-related applications that may target women as primary consumers were noted by G. B. Akar. 3D virtual malls were described as a game-like environment where you can navigate through shops. A 3D dressup simulator would allow selected garments to be mixed and matched on an avatar and viewed from different angles. Finally, the use of 3D in telemarketing was noted as an enhancement that might make home shopping more attractive.

Akar also suggested that application of 3DTV in the areas of professional and vocational training (for instance, to become a surgeon, pilot, or technician) has the potential to benefit women particularly, because some studies seem to show that women are more inclined towards visual learning. She added that a similar potential exists in K-12 education, especially in subjects such as geometry, chemistry, biology, and physics where visualization or simulation of complex structures or phenomena are vital. This may also help increase the interest of women in science and engineering.

Will it be important for companies to target one or the other gender to sell 3DTV consumer equipment or applications?

A. Boev and G. Ziegler mentioned market studies on which gender dominated in the decision to purchase consumer electronics, and which features were decisive (technical parameters, design, etc.). Furthermore some studies seem to indicate sex differences in perception and spatial ability, which may have implications especially on immersive technologies. Given that these issues are highly charged and there are many unresolved claims, it is difficult to make any meaningful conclusions.

\section{Acknowledgments}

We would like to thank the following participants of the Network of Excellence for their contributions to the discussions which formed the basis of this work: Gozde B. Akar (Middle East Technical University, Ankara, Turkey), Atanas Boev (Tampere University of Technology, Tampere, Finland), Reha Civanlar (Koç University, Istanbul, Turkey, now DoCoMo Labs, Palo Alto, USA), Sven Fleck (University of Tübingen, Tübingen, Germany), Rossitza Ilieva (Bilkent University, Ankara, Turkey), Matthias Kautzner (Fraunhofer Institute for Telecommunications/Heinrich-Hertz-Institut, Berlin, Germany), Kıvanç Köse (Bilkent University, Ankara, Turkey), Matthias Kunter (Technical University of Berlin, Berlin, Germany), Haldun M. Ozaktas (Bilkent University, Ankara, Turkey), Mehmet Özkan (Momentum AŞ, Istanbul, Turkey), Ismo Rakkolainen (FogScreen Inc., Helsinki, Finland), Simeon Sainov (Bulgarian Academy of Sciences, Sofia, Bulgaria), Vaclav Skala (University of West Bohemia in Plzen, Plzen, Czech Republic), Aljoscha Smolic (Fraunhofer 
Institute for Telecommunications/Heinrich-Hertz-Institut, Berlin, Germany), Nikolce Stefanoski (University of Hannover, Hannover, Germany), Philip Surman (De Montfort University, Leicester, United Kingdom), Cemil Türün (Yogurt Technologies Ltd., Istanbul, Turkey), Yasemin Yardımcı (Middle East Technical University, Ankara, Turkey), Ali Özgür Yöntem (Bilkent University, Ankara, Turkey), Gernot Ziegler (Max Planck Institute for Informatics, Saarbrücken, Germany).

We are especially grateful to the following external participants for their contributions to the discussions: Güliz Ger (Bilkent University, Faculty of Business Administration, Department of Management, Ankara, Turkey), an expert on the sociocultural dimensions of consumption, consumption and marketing in transitional societies and groups, and related issues of globalization, modernity, and tradition; Murat Karamüftüoğlu (Bilkent University, Faculty of Art, Design, and Architecture, Department of Communication and Design, Ankara, Turkey), an expert on information retrieval theory, design, and evaluation, computer mediated communication and collaborative work, computer semiotics, philosophical foundations of information systems, and the organizational, social and political implications of information systems; Dilek Kaya-Mutlu (Bilkent University, Faculty of Art, Design, and Architecture, Department of Graphic Design, Ankara, Turkey), an expert on film studies with an emphasis on audience studies, film reception, and Turkish cinema; Jinwoong Kim (ETRI, Radio and Broadcasting Research Laboratory, Daejeon, Republic of Korea), 3DTV Project Leader; Fatih Porikli (Mitsubishi Electric Research Labs, Cambridge, USA), Principal Member and Computer Vision Technical Leader; Özlem Sandıkçı (Bilkent University, Faculty of Business Administration, Department of Management, Ankara, Turkey), an expert on culturally oriented issues in marketing, including advertising reception, gender and advertising, consumption culture, and the relationships between modernity, postmodernity, globalization and consumption. Special thanks goes to Gozde B. Akar (Middle East Technical University, Faculty of Engineering, Department of Electrical and Electronics Engineering, Ankara, Turkey) and Yasemin Soysal (University of Essex, Department of Sociology, Colchester, United Kingdom) for their critical comments on Part IV.

We would like to take this opportunity to also thank Levent Onural of Bilkent University for his support as leader of the Network of Excellence on Three-Dimensional Television. Finally, we are grateful to Kirsten Ward for careful editing of the manuscript.

This work is supported by the EC within FP6 under Grant 511568 with the acronym 3DTV. 